\title{
RESIDUAL GAS PRESSURE PROFILE IN THE RECYCLER RING
}

\author{
K. Gounder *, J. Marriner, S. Mishra, and Terry Anderson.. \\ FNAL, Batavia, IL 60510, USA
}

\section{Abstract}

We simulate the pressure profile of residual gases from basic principles using detailed beam pipe geometry and the relevant physical parameters. These profiles are compared with the actual ion gauge measurements and is being used to predict the vacuum contribution to the Recycler Ring beam lifetime. Work supported by the U.S. Department of Energy under contract No. DE-AC02-76CH03000.

\section{INTRODUCTION}

The Recycler Ring [1] located in the upper portion of the Main Injector tunnel at Fermilab is designed as a storage ring for antiprotons. Antiprotons transferred from the Accumulator and the residual Tevatron stores will be cooled and stored in the Recycler before reinjection into the Tevatron. The stacking rate of the antiprotons in the Accumulator is improved by steady transfer of antiprotons to the Recycler Ring when the stack size becomes sufficiently large. Thus, as a part of Run II upgrade, the Recycler Ring will provide a factor of 2-3 improvement in luminosity. Pressently, the Recycler Ring (RR) is being commissioned using protons as well as antiprotons. Here we describe the design of the RR vacuum, compute the pressure profile for the residual vacuum gases around the ring and compare with measured partial pressures of residual gases. The simulated partial pressures of gases can be used to estimate the RR beam life time and emittance growth due to the interaction of residual gases with the beam particles. The relevant RR parameters used are listed in Table 1. More detailed description of the Recycler Ring can be found elsewhere [1].

Table 1: Recycler Ring Paramaters

\begin{tabular}{|l|c|}
\hline Paramater & Value \\
\hline Acceptance $(\mathrm{mm}-\mathrm{mr})$ & $40.0 \pi$ \\
Average $\beta(\mathrm{m})$ & 40.0 \\
Average beam pipe radius (in $\mathrm{m})$ & 0.023 \\
Beam energy $(\mathrm{GeV})$ & 8.89 \\
Average beam $\beta$ & 0.998 \\
Average beam $\gamma$ & 9.48 \\
Maximum energy loss $(\mathrm{GeV})$ & 0.089 \\
\hline
\end{tabular}

\section{RECYCLER RING VACUUM SYSTEM}

The RR vacuum design was motivated by the requirement of storing about $2.5 \times 10^{12}$ antiprotons with less

\footnotetext{
*gounder@fnal.gov
}

than 5\% loss for stores of duration 8 hours or more. This requires a beam life time of greater than 100 hours and a transverse emittance growth rate less than $2 \pi \mathrm{mm}$ $\mathrm{mr} /$ hour. In turn, this imposes very stringent requirements on the amount of residual gases allowed in the RR vacuum. Therefore the vacuum design is expected to support only a few tenths of nano Torr of total gas pressure with only a minute amount of heavy gases such as Argon, $\mathrm{CO}_{2}$ etc..

The RR beam pipe is made up of stainless steel and is mostly elliptical in shape with horizontal major axis 9.67 $\mathrm{cm}$ and vertical minor axis $4.44 \mathrm{~cm}$. It also has 3 and 4 inch radii circular portions (for about $7 \%$ of the total length) in the straight sections of the ring. There are over 640 Titanium Sublimation Pumps (TSP) distributed periodically around the Ring with a distance of $4-5 \mathrm{~m}$ between the pumps depending on the location. These were custom designed and built by Fermilab with a maximum pumping speed of 10460 liters/second for Nitrogen. These are fired manually every 6-12 months depending on the gas pressure needs. There are also 226 Ion Pumps (IP) located around the Ring most of them fitted directly to a TSP. A fraction of the Ion pumps are specifically located around special devices such as Lambertson magnets, Schottky detectors and stochastic cooling tanks etc. All the Ion pumps are diode pumps made by Varian [3] and a large fraction of them have a Nitrogen pumping speed of 30 liters/second. The rest are specifically modified to pump noble gases such as Argon more efficiently. The details of the beam pipe geometry and vacuum pump specifications are provided in Table 2 .

The vacuum pressure is readout and monitored by 30 Ion gauges along with ion pumps located through out the Ring. There are also 8 Residual gas analyzers (RGA) installed at strategic locations providing partial pressures of residual gases present. The schematics of the pump configuration is shown in the figure below. The RR vacuum is divided into 30 sections separated by control valves. This configuration helps to isolate parts of the vacuum when necessary during maintenance, repairs, leaks or installation of new devices.

\section{RECYCLER RING VACUUM RESIDUAL GASES}

The RR ultra high vacuum is maintained by arrays of TSPs and ion pumps has a total pressure of a fraction of a nano Torr. The types of gases present in the vacuum and their partial pressures can be obtained by RGA measurements done often around the ring. The major constitutents of the vacuum (average of RGA readings) are listed in Table 3 . We have also detected some very minor quantities of hydro carbons such as Ethane, Etheylene etc. 


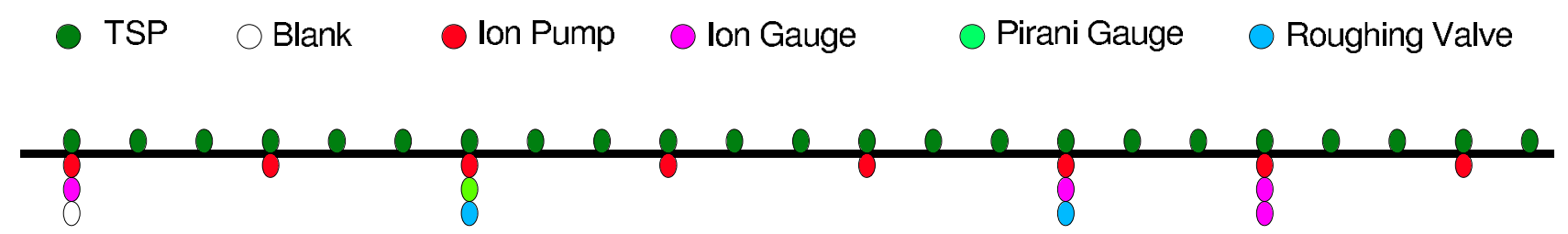

Figure 1: The Recycler Ring Vacuum pump configuration for major portions of the Ring. The remaining portions are being upgraded to have the same configuration. Besides the periodic array of pumps, there are special ion pumps and TSPs installed at critical locations such as Lambertson magnets, stochastic cooling tanks, Schottky detectors etc.

\begin{tabular}{|l|c|}
\hline \multicolumn{1}{|c|}{ Parameter } & Value \\
\hline \hline Vacuum Temperature & $293 \mathrm{~K}$ \\
TSP Pumping Efficiency & $\approx 0.05$ \\
Ion Pump Efficiency & 1.0 \\
Ring Length [m] & 3319.4 \\
Number of TSPs & 644 \\
Number of Ion Pumps & 226 \\
Number of Ion Gauges & 30 \\
Number of Vacuum Sectors & 30 \\
Number of RGAs & 8 \\
\hline \hline Elliptical Sections & {$[$ MKS Units] } \\
\hline Major axis [m] & $9.667 \times 10^{-2}$ \\
Minor axis [m] & $4.444 \times 10^{-2}$ \\
Area of TSP Aperture [m $\left.{ }^{2}\right]$ & $34.90 \times 10^{-4}$ \\
Area of Ion Pump Aperture [m $\left.{ }^{2}\right]$ & $28.60 \times 10^{-4}$ \\
Length of Ion Pump [m] & $6.033 \times 10^{-2}$ \\
\hline \hline Circular Sections & {$[$ MKS Units] } \\
\hline Diameter of 3' Beam Pipe [m] & $7.303 \times 10^{-2}$ \\
Diameter of 4' Beam Pipe [m] & $9.8425 \times 10^{-2}$ \\
\hline
\end{tabular}

Table 2: Beam pipe geometry and vacuum pump data for the Recycler Ring.

\section{PRESSURE PROFILE SIMULATION}

Knowing the complete beam pipe geometry, the vacuum pump configuration and the details of gas parameters such as outgassing rates, conductances etc., we can simulate the pressure profile of each gas around the ring. For simulation purposes, we treat the 'unknown' component as Nitrogen. The TSPs do not contribute to pumping out Argon or Methane while pumping other gases such as Hydrogen, Water, Carbon Monoxide, Carbon Dioxide and Nitrogen. The ion pumps pump all gases including Argon and hydro carbons such as Ethelyne, Methane etc.

The vacuum pressure $P$, the pumping speed $s$, the conductance of the beam pipe $\mathrm{c}$ and the outgassing rate $\mathrm{q}$ at a given location are related by:

$$
\frac{d}{d z}\left\{c \frac{d P}{d z}\right\}-s P+q=0
$$

A technique based on the approximation of finite differences is used to solve the above differential equation [4]. This method has the advantage of being numerically stable

\begin{tabular}{|l|c|c|c|}
\hline \multicolumn{1}{|c|}{ Gas } & $\begin{array}{c}\text { Pump. Speed } \\
\text { [liter/s] }\end{array}$ & $\begin{array}{c}\text { Outgassing Rate } \\
\left.\text { [(nTorr.liter)/(s } \mathrm{m}^{2}\right)\end{array}$ & $\begin{array}{c}\text { Avg. Press. } \\
\text { [nTorr] }\end{array}$ \\
\hline \hline $\mathrm{H}_{2}$ & 3490.000 & 4.500 & 0.354 \\
\hline $\mathrm{H}_{2} \mathrm{O}$ & 3490.000 & 0.290 & 0.038 \\
\hline $\mathrm{CO}$ & 10460.000 & 0.190 & 0.019 \\
\hline $\mathrm{CO}_{2}$ & 8775.000 & 0.110 & 0.015 \\
\hline $\mathrm{CH}_{4}$ & 30.000 & 0.003 & 0.005 \\
\hline $\mathrm{N}_{2}$ & 10460.000 & 0.081 & 0.105 \\
\hline $\mathrm{Ar}$ & 1.700 & 0.001 & 0.001 \\
\hline Total & & 5.185 & 0.537 \\
\hline
\end{tabular}

Table 3: The relevant gas parameters required for simulation of the pressure profile around the ring. The "unknown component' of gases is treated as Nitrogen.

when the system is very long such as the Recycler Ring. We cast the first term as:

$$
\begin{gathered}
\frac{d}{d z}\left\{c_{i} \frac{d P}{d z}\right\}=\frac{\left(c_{i+1}+c_{i}\right) P_{i+1}+\left(c_{I}+c_{i-1}\right) P_{i-1}}{2 \Delta z^{2}} \\
-\frac{\left(c_{i+1}+c_{i-1}+2 c_{i}\right) P_{i}}{2 \Delta z^{2}}
\end{gathered}
$$

The above equation becomes:

$$
\begin{gathered}
\left\{\frac{-\left(c_{i+1}+c_{i-1}+2 c_{i}\right)}{2}-s_{i} \Delta z^{2}\right\} P_{i}+\frac{c_{i}+c_{i+1}}{2} P_{i+1} \\
+\frac{c_{i}+c_{i-1}}{2} P_{i-1}=q_{i} \Delta z^{2}
\end{gathered}
$$

The boundary conditions at each end of the segment should be specified:

$$
Q_{i}=-c_{i} \frac{P_{I+1}-P_{i-1}}{2 \Delta z}
$$

This allows us to solve for either $P_{i+1}$ or $P_{i-1}$ in terms of the flow in the ith segment. In fact we can form a tridiagonal matrix as shown below. This system of equations can be solved by Gaussian elimination technique and back substitution. A simpler alternative approach for an array of pumps was used to verify the numerical accuracy of this method.

For each contitutent gas, the pressure profile around the ring has been simulated. The results for Hydrogen and 
Methane are shown in Figure 2 for region in the 400

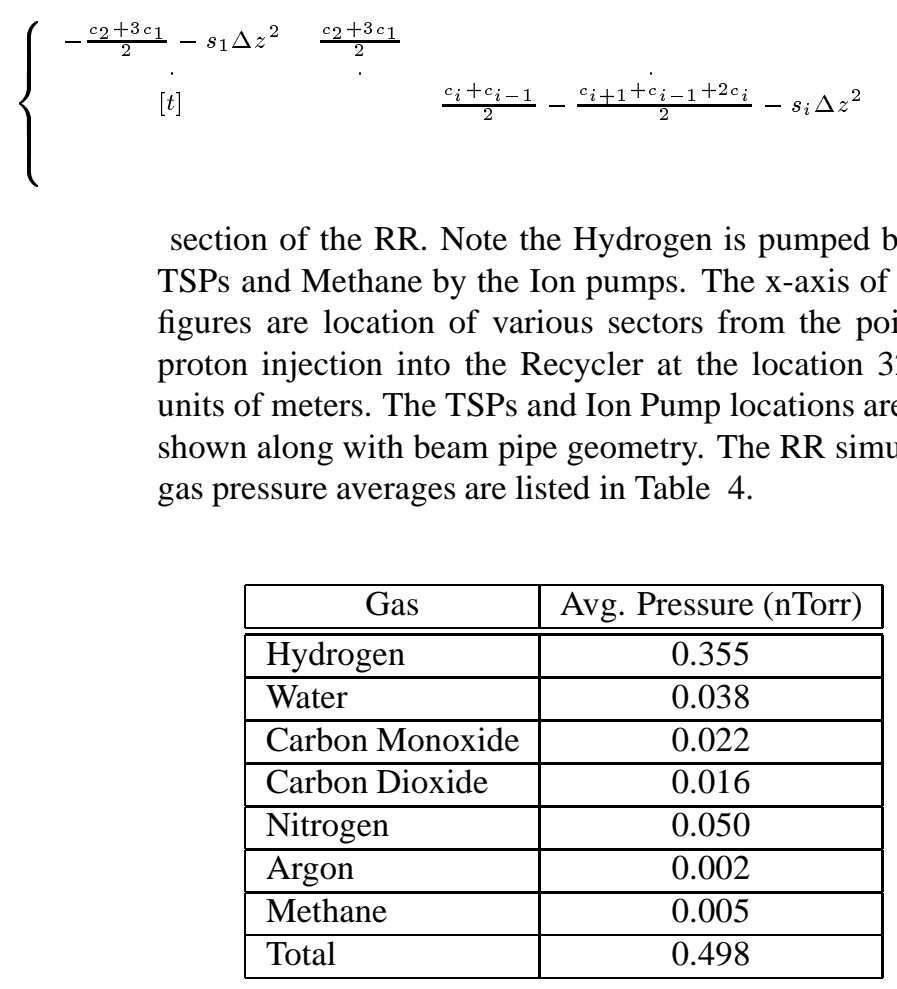

Table 4: The Ring wide average of the simulated pressure of residual gases. The 'unknown' gas component is treated as Nitrogen.

\section{COMPARISON WITH MEASUREMENTS}

The ring wide average partial pressures shown in Table 3 were obtained from RGA measurements and nearby Ion Gauge readings (normalization) taken at 7 different locations around the ring. The raw RGA/IG readings were corrected for each gas for calibration, conductance of the connecting assemblies as well as the measurement location with respect to the relevant pump locations. On the other hand, the simulated values shown in Table 4 greatly depends on knowledge of the beam pipe geometry, outgassing rates measured in mockups and pumping efficiencies of TSPs (time varying) and Ion pumps. To obtain predictive power for the simulation techniques, we have adjusted the outgassing rates to reasonably produce the observed partial pressures. This is evident by comparing Tables 3 and 4 . The partial pressures of residual gases can be used to determine the contribution of beam-gas scattering for the RR beam lifetime [5].

\section{REFERENCES}

[1] Gerry Jackson, “The Fermilab Recycler Ring Technical Design Report”, November 1996, Fermilab-TM-1991; Mishra, "Status of Fermilab Recycler Ring", EPAC2002, Paris, June 2002.
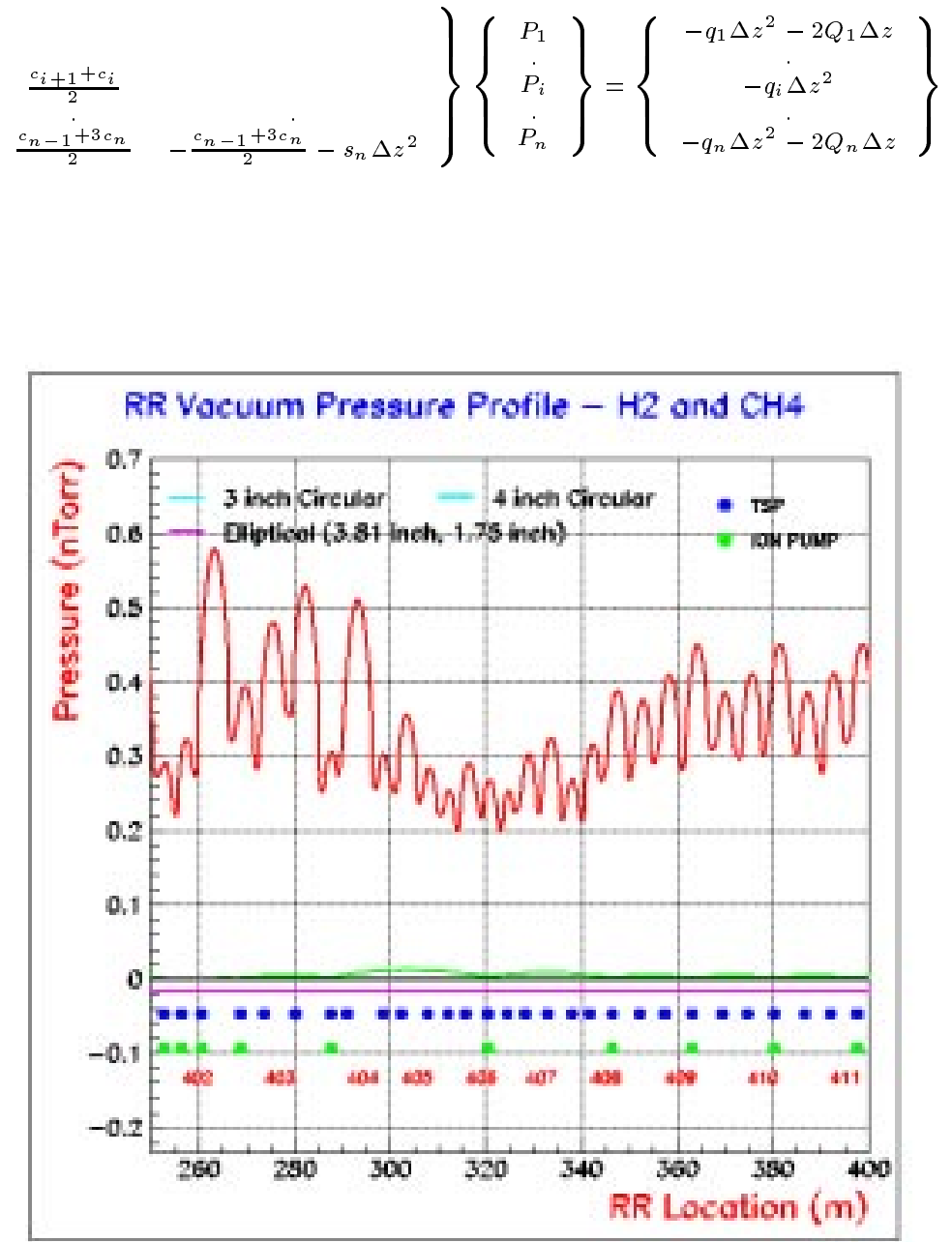

Figure 2: The simulated pressure profiles for Hydrogen (red - upper) and Methane (green - lower) are shown for region in the 400 section of the RR. Note the Hydrogen is pumped by the TSPs and Methane by the Ion pumps. The $\mathrm{x}$-axis are location of various sectors from the point of proton injection into the Recycler at the location 328 in units of meters. The TSPs and Ion Pump locations are also shown along with beam pipe geometry.

[2] J. Marriner et. al., "Run II Handbook", at http://wwwbd.fnal.gov/runII/index.html; Also see K. Gounder, ”The Status of Run II at Fermilab", in the 2002 proceedings of ICHEP, Amsterdam, July 2002.

[3] Varian Vacuum Products Lexington, 121 Hartwell Avenue, Massachusetts, 02173 USA.

[4] M. K. Sullivan, "A Method for Calculating Pressure Profiles in Vacuum Pipes", SLAC, 1993.

[5] K. Gounder et al., "Recycler Ring Beam Lifetime", FermilabConf-01-186-E, PAC-2001-RPPH055, July 2001. 